\title{
Phonetic symbolism and feeling of knowing
}

\author{
ASHER KORIAT \\ Hebrew University, Jerusalem, Israel
}

\begin{abstract}
American subjects matched English antonym pairs with antonym pairs from Thai, Kannada, and Yoruba. For each match they also indicated their degree of feeling of knowing (FOK). Although translation accuracy was in general better than chance, suggesting universal phonetic symbolism, it was found to vary considerably with the subject's FOK ratings: Translations evoking stronger FOK were more likely to be correct than translations evoking weaker FOK, and matches which proved correct were assigned higher FOK ratings than matches which proved incorrect. Several implications of the finding that subjects can monitor their success in guessing the meaning of words from foreign languages are outlined.
\end{abstract}

One intriguing feature of human cognition is that in searching his memory for a solicited item an individual can often accurately evaluate whether or not the item retrieved is indeed the appropriate one. The capacity to know that one knows, apparently indispensable for effective functioning of the memory system, is amply demonstrated by everyday experience. Only recently, however, has it become a subject for experimental investigation. Subjects unable to recall a solicited item were found to predict accurately whe ther they would be able to recognize that item if presented among distractors (Blake, 1973; Hart, 1965, 1967). Murdock (1966), using a recall task, found the probability of correct recall positively correlated with the subject's confidence ratings. A similar relationship was observed by Tulving and Thomson (1971) for recognition memory. Thus, it appears that the individual's subjective confidence is predictive of his actual memory performance.

The purpose of the present study is to examine the possibility that ratings of feeling of knowing (FOK) bear a similar relationship to accuracy of performance in a phonetic symbolism task which requires matching the words in a noncognate foreign language with their English equivalents. The idea of a universal phonetic symbolism implies that sounds tend to have intrinsic symbolic connotations which are universally shared by all humans and that traces of these sound-meaning linkages survive in all natural languages. If the universally shared connotations of sounds are assumed to be stored in memory in a manner analogous to that in which learned information is stored, the individual's rating of FOK might also be correlated with accuracy of performance in a task requiring the guessing of the meaning of foreign words.

Although the possibility of a universal phonetic symbolism is by no means proved, there is some evidence that with certain procedural confinements

The present investigation was supported by a grant from the Faculty of Social Sciences, Hebrew University of Jerusalem. I wish to thank Helen Kubnik for her help in gathering the data and Dan Slobin for his kind help in providing the stimulus list. people can be shown to guess the meaning of foreign words with better than chance success (Hörman, 1971). Perhaps the strongest support for the hypothesis of universal phonetic symbolism has been obtained in studies employing a word-matching technique where English speakers are asked to match two antonyms in a noncognate language with their English equivalents (Ervin-Tripp \& Slobin, 1966; Slobin, 1968). Taylor and Taylor (1965) criticized word-matching studies on several grounds, although they themselves are willing to admit the possibility of a culturally delimited phonetic symbolism, i.e., a common conception of sound-meaning relationships among speakers of a given language.

The degree of subjective confidence associated with the endorsement of a particular guess is one datum which has never been exploited in the study of phonetic symbolism. It has been the impression of the present author that some of the items in a word-matching task are responded to with great ease and strong confidence, as though the appropriate match feels intuitively self-evident, and that with such items the response is more likely to be correct than wrong. Further, when subjects who agree that a correct match is intuitively obvious are asked to deliberate on the grounds for their response, they often provide quite diverse reasons, as though they all feel the same response to be self-evident but for different reasons.

The present study examines the relationship between feeling of knowing and accuracy of translation in a word-matching task. It is predicted that (a) correct matchings will be associated with stronger FOK ratings than incorrect matchings, and (b) the FOK rating associated with a given translation will be predictive of the accuracy of that translation: The stronger the FOK rating, the higher the likelihood that the match will be correct.

\section{METHOD}

\section{Stimulus Materials}

The list employed in this work is that used by Slobin (1968) in his study of phonetic symbolism. It consists of antonym pairs matched with the correspondent translations in Thai, Kanasese 
Table 1

Frequencies of Correct and Incorrect Translations for Responses With Different FOK Ratings

\begin{tabular}{ccccc}
$\begin{array}{c}\text { FOK } \\
\text { Rating }\end{array}$ & Incorrect & Correct & Total & $\begin{array}{c}\text { Percentage } \\
\text { Correct }\end{array}$ \\
\hline 1 & 292 & 334 & 626 & 53.35 \\
2 & 427 & 508 & 935 & 54.33 \\
3 & 347 & 524 & 871 & 60.16 \\
4 & 201 & 392 & 593 & 66.10 \\
Total & 1267 & 1758 & 3025 & 58.12 \\
\hline
\end{tabular}

(or Kannada), and Yoruba. The pairs were chosen to represent the three major dimensions of the semantic differential and include both sensible and nonsensible continua. Over all three foreign languages there were 56 such pairs. A visual presentation was employed. All the material was compiled in a booklet containing the instructions and the antonym pairs. Each English pair was matched by its corresponding pair in the following format:

$\begin{array}{ll}\text { deep } & \text { tuun } \\ \text { shallow } & \text { luk }\end{array}$

The order of the words in each foreign pair was determined on a random basis. The list of pairs from each language was presented as a block. The construction of the list is more fully described by Slobin (1968).

\section{Subjects and Procedure}

The written instructions directed subjects to indicate the appropriate match for each of the items and to rate on a 4-point scale the subjective confidence associated with each match. The instructions concerned with the confidence ratings read as follows:

"I am also interested in your confidence in the correctness of your choice. Although your answer is based only on guessing, in some case more than in others, after making a choice you have the unexplainable feeling that you may be correct. It is this elusive feeling that we ask you to try to monitor. After marking an answer to each item, try to judge, even by guessing, the tikelihood of your having answered correctly. Indicate your judgment on the scale after each pair.

"Mark 1 when you feel your answer is based on a totally wild guess. Ideally, we should expect you to rate the $25 \%$ of your answers about which you feel least confident with a 1 .

"Mark 4 when you feel your answer is reasonably likely to be right. Ideally, we should expect you to rate the $25 \%$ of your answers about which you feel most confident with a 4 .

"Numbers 2 and 3 represent confidence ratings between 1 and 4. Although you may feel now that you will want to rate all of your answers with a 1 , your success in choosing language pairs correctly will actually be greater than you anticipate. We ask you here only to pay very close attention to your feelings while marking each choice and to make use of each rating at least a few times."

Fifty-five students enrolled at the Hebrew University, whose native language was English, participated in the study. There were 29 males and 26 females. The experiment was conducted in small groups.

\section{RESULTS}

We will first examine the accuracy of translation. One item had to be eliminated from the analysis due to a typographical error. For the remaining 55 items the mean perceptage of correct translation was 58.12 , which is significantly better than chance at the .001 level $(\mathrm{t}=9.03, \mathrm{df}=54)$.

In order for a pair translation to be different from chance at the .01 level, it must be made by at least $67 \%$ of the subjects. Using this criterion, 21 items yielded a significantly correct matching and 7 items yielded a significantly incorrect matching. Of the 55 subjects who participated, 49 subjects matched correctly more than $50 \%$ of the pairs, the remaining 6 subjects demonstrating close to but telow $50 \%$.

On the whole, the results appear to replicate those obtained by Slobin (1968) in showing that subjects can guess the meaning of noncognate words with better than chance success.

We turn now to examine the hypothesis that likelihood of correct matching varies positively with degree of subjective confidence. Table 1 presents perhaps the most convenient summary of the data pertaining to this hypothesis. For each subject, each of the pair translations was classified in terms of both the correctness of the translation and the FOK rating associated with it. Table 1 shows the contingency frequencies pooled across items and individuals. It can be observed that the percentage of correct translations increases monotonically with degree of FOK rating. A chi-square analysis is not appropriate to evaluate the data of Table 1 due to the dependencies involved. It should be noted, however, that the correct percentages associated with the four levels of FOK ratings are ranked exactly as predicted and the probability of this event's occurrence by chance is less than .05 .

When all responses are combined, the contributions to the total effects of individual pairs and of individual subjects are unequal. Since the hypothesis regarding the relationship between FOK and accuracy of performance implies essentially an intra-individual correlation, a better way to evaluate it is to obtain summary scores which reflect such intra-individual contingencies. For each subject, four translation accuracy scores were calculated consisting of the percentages of correct translations for all items that he assigned FOK ratings of $1,2,3$, and 4 , respectively. The means of these accuracy scores over all subjects were $53.57,53.90,60.23$, and 65.93 , respectively. The last average is based on 52 subjects, since 3 subjects never employed an FOK rating of 4. A one-way repeated analysis of variance based on the 52 subjects yielded $F(3,51)=5.741$, which is significant at the 001 level.

Since individuals displayed a great variability in the relative utilization of the four FOK ratings, some of the individual translation accuracy scores were based on as few as a single item. Since it also appears that the major difference in accuracy of translation obtains between FOK Levels 1 and 2, on the one hand, and FOK Levels 3 and 4, on the other, it seems appropriate to regroup the FOK categories into weak FOK (Categories 1 and 2) and strong FOK (Categories 3 and 4). Individual correct 
Table 2

Summary of Data on the Relationship Between Translation Accuracy and FOK Ratings by Semantic Categories

\begin{tabular}{|c|c|c|c|c|c|c|c|}
\hline \multirow[b]{2}{*}{ Category } & \multirow[b]{2}{*}{$\begin{array}{l}\text { Number } \\
\text { of Pairs }\end{array}$} & \multicolumn{3}{|c|}{$\begin{array}{c}\text { Mean Percentage Correct* } \\
\text { Responses }\end{array}$} & \multicolumn{3}{|c|}{$\begin{array}{c}\text { Mean FOK Rating } \\
\text { Responses }\end{array}$} \\
\hline & & $\begin{array}{l}\text { Weak } \\
\text { FOK }\end{array}$ & $\begin{array}{c}\text { Strong } \\
\text { FOK }\end{array}$ & All & $\begin{array}{l}\text { Weak } \\
\text { FOK }\end{array}$ & $\begin{array}{c}\text { Strong } \\
\text { FOK }\end{array}$ & All \\
\hline Nonsensible & 20 & 54.69 & 69.05 & 61.82 & 2.28 & 2.62 & 2.49 \\
\hline Sensible & 35 & 53.53 & 58.71 & 56.00 & 2.40 & 2.51 & 2.46 \\
\hline Magnitude & 18 & 56.47 & 60.21 & 58.28 & 2.45 & 2.52 & 2.49 \\
\hline Nonmagnitude & 17 & 50.50 & 57.08 & 53.58 & 2.36 & 2.51 & 2.44 \\
\hline Evaluative & 19 & 54.48 & 64.08 & 58.85 & 2.24 & 2.48 & 2.39 \\
\hline Potency & 18 & 53.19 & 64.27 & 58.48 & 2.33 & 2.57 & 2.47 \\
\hline Activity & 18 & 54.11 & 59.61 & 56.97 & 2.51 & 2.62 & 2.57 \\
\hline
\end{tabular}

*Calculated over both individuals and iterns.

percentage scores were computed for weak FOK and strong FOK responses. Four subjects who assigned ratings 3 and 4 to fewer than 10 items were eliminated. For the remaining subjects, the mean correct percentage obtained for weak FOK responses was 53.79 , compared to 62.30 for strong FOK responses, the difference being highly significant $(t=4.45, d f=51, p<.001)$. A comparison of the mean correct percentage obtaired for weak FOK responses to the mean expected by chance $(50 \%)$ yields $t=2.55$, which approaches but does not reach the .01 level of significance.

A related question is whether subjects are better able to monitor their guesses by assigning strong FOK ratings to matches which prove correct than to matches which prove incorrect. The subjects assigned an average FOK rating of 2.37 to their incorrect responses and an average of 2.56 to their correct responses. A $t$ test comparison yielded $t=4.35$, significant at the .001 level.

There were consistent individual differences in the tendency to assign relatively high or relatively low FOK ratings. These differences appear to reflect personal standards or response sets; therefore, it is not surprising that individuals who assigned a relatively high average FOK rating did not demonstrate generally superior translation accuracy. On the other hand, items which were correctly translated by a larger number of subjects tended to elicit stronger FOK ratings on the average. The correlation across items between percentage of correct translation and mean FOK ratings was .41 . The 37 items answered correctly by over $50 \%$ of the subjects were associated with a mean FOK rating of 2.55 , and the 18 items incorrectly translated by over $50 \%$ of the subjects were associated with a mean FOK rating of 2.31 . A t test comparison yielded $t=6.82$, significant beyond the .001 level.

Slobin (1968) examined in his study the generality of phonetic symbolism over several semantic domains. For this purpose, he classified the various antonym pairs into several categories. The same classification was employed in the present study to examine the generality of the confidence-accuracy correlation. Table 2 presents some pertinent data. It can be seen that for all semantic categories percentage of correct translations is higher for strong FOK responses than for weak FOK responses. Similarly, for all semantic categories correct translations are associated with higher FOK ratings than incorrect translations. It thus appears that the confidence-association relationship is not restricted to terms denoting magnitude and its common associates, nor to sensible terms alone. The extent of this relationship, however, seems to vary from one category to another, but it would be premature to deliberate on the reasons for these differences before they prove reliable.

\section{DISCUSSION}

The results of this investigation suggest that, in the context of phonetic symbolism, objective knowing and subjective knowing are correlated. It was demonstrated that (a) the stronger the feeling of knowing associated with a guess regarding the meaning of a noncognate foreign word, the greater the likelihood that the guess is accurate; and (b) guesses which prove correct tend to evoke stronger FOK ratings than guesses which prove incorrect.

It was shown further that an overall analysis of the accuracy data supported the contention that subjects can guess the meaning of foreign words with better than chance success. In addition, an analysis which takes into account FOK ratings showed that this is even more strongly true for responses associated with high FOK ratings. Guesses associated with low FOK ratings tended to be hardly more correct than chance.

One methodological question raised by these results concerns the appropriate index to be used in assessing the existence of a universal phonetic symbolism. Taylor and Taylor (1965), criticizing current word-matching experiments, noted that "no study since Tsuru and Fries [1933] has reported a list matched better than 65\% correct overall, and only 3 of 21 lists have given over $60 \%$." These experiments, they concluded, cannot be considered as supportive of the idea of a universal phonetic symbolism. In the present study, however, correct translation was found to be as low as $53 \%$ and as high as $66 \%$, depending on the level of FOK rating 
involved. One question suggested by these results is whether or not confidence ratings should be taken into account in the evaluation of phonetic symbolism. Thus, it could be argued that phonetic symbolism is best manifest in those responses which the individual is willing to endorse with strong confidence, not in those responses which he is forced to make on the basis of what he considers to be a wild guess. According to this view, the more meaningful index is represented by the $66 \%$ correct performance, which strongly supports the notion of a universal phonetic symbolism. Clearly, whether or not one decides to take FOK ratings into account depends on one's theoretical assumptions. All that our results suggest is that this decision might prove crucial in determining one's conclusions.

The substantial implications of the results are harder to evaluate at this point. On the one hand, the confidence-accuracy correlation, taken in conjunction with the finding that overall success of translation is significantly better than chance, appears on the face of it to be particularly consonant with a conception which postulates intrinsic, universally shared symbolisms. Traditionally, the concept of "self-evidence" has occupied a central position in intuitionistic theories which accept the notion of a priori ultimate truths that are directly or intuitively apprehended (Westcott, 1968). In these theories, universality and self-evidence are assumed to go hand in hand, being conjoint characteristics of innate cognitions. Thus, the confidence-accuracy association observed in the present study would be interpreted by such theories as reflecting a basic property of human cognition, namely, that universally shared notions are endowed with the capacity to present themselves as self-evident to the individual's awareness. On the other hand, however, those who do not accept the idea that the symbolic connotations of sound reflect innate, universally shared sound-meaning linkages (Brown, 1958; Taylor, 1963) would not necessarily regard the confidence-accuracy correlation, in itself, as detrimental to their position.
This correlation, they would claim, might indicate something about the manner in which the conception of the symbolic connotation of sounds is stored in memory but has little bearing on the issue of the origin of this conception.

Although more definite conclusions should await further research, in the view of the present author the results on the whole can best be taken to suggest the following. First, universal phonetic symbolism is partial in the sense that (a) traces of it exist only in some of the words of a natural language, and (b) certain sounds or sound combinations do not possess symbolic connotations with respect to one or more dimensions of meaning. Second, the degree to which a certain sound possesses symbolic connotations with regard to a particular dimension of meaning is available to the individual's awareness.

\section{REFERENCES}

Blake, M. Prediction of recognition when recall fails: Exploring the feeling of knowing phenomenon. Journal of Verbal Learning and Verbal Behavior, 1973, 12, 311.319.

Brown, R. Words and things. Glencoe, Ill: Free Press, 1958.

Ervin-Tripp, S. M., \& Slobin, D. I. Psy cholinguistics. Annual Review of Psy chology, 1966, 17, 435-474.

Hart, J. T. Memory and the feeling of knowing experience. Joumal of Educational Psy chology, 1965, 56, 208-216.

Hart, J. T. Memory and the memory monitoring process. Journal of Verbal Learning and Verbal Behavior, 1967, 6, 685-691.

Hörman, H. Psycholinguistics: An introduction to research and theory. New York: Springer-Verlag, 1971.

Murdock, B. B. The criterion problem in short-term memory. Journal of Experimental Psy chology, 1966, 72, 317-324.

Slobin, D. Antonymic phonetic symbolism in three natural languages. Journal of Personality and Social Psychology, $1968,10,301-305$.

Taylor, I. K. Phonetic symbosis re-examined. Psychological Bulletin, 1963, 60, 200-209.

Taylor, 1. K., \& Taylor, M. M. Another look at phonetic sy mbolism. Psychological Bulletin, 1965, 64, 413-427.

Tsuru, S., \& Fries, H. S. A problem in meaning. Journal of General Psy chology, 1933, 8, 281-284.

Tulving, E., \& Thomson, D. M. Retrieval processes in recognition memory: Effects of associative context. Joumal of Experimental Psy chology, 1971, 87, 116-124.

Westcott, M. R. Toward a contemporary psychology of intuition. New York: Holt, Rinehart \& Winston, 1968. 